\title{
A REMARK ON SINGULAR INTEGRALS WITH COMPLEX HOMOGENEITY
}

\author{
ROGER L. JONES
}

(Communicated by J. Marshall Ash)

\begin{abstract}
The Hilbert transform can be approximated by operators with Fourier multiplier given by $c_{\gamma} \operatorname{sgn}(\xi)|\xi|^{i \gamma}$. If we let $\gamma \rightarrow 0$ it is known that these operators converge to the Hilbert transform in $L^{2}$ norm. It has also been shown that as $\gamma \rightarrow 0$ these operators may diverge even for functions in $L^{2}$. In this paper it is shown that we also will have divergence if we select any sequence $\left\{\gamma_{n}\right\}_{n=1}^{\infty}$ such that $\gamma_{n} \rightarrow 0$. The proof makes use of Bourgain's entropy method.
\end{abstract}

\section{INTRODUCTION}

Let $f$ be an integrable function on the real line $\mathbb{R}$. The Hilbert transform $\tilde{f}$ is the principal value convolution of $f$ with the singular kernel $\frac{1}{\pi t}$. At almost every point, the integral defining $\tilde{f}$ coinverges, and hence $\tilde{f}$ exists. The existence of $\tilde{f}$ is a central fact in the theory of real and complex analysis, and one which has been generalized in a variety of directions. In the 1950s a possible new approach to establishing the existence of this important operator was suggested. In one dimension the idea was to replace convolution with $\frac{1}{\pi t}$ by convolution with $\frac{1}{\pi t}|t|^{-i \gamma}$. The hope was that this family of operators would be easier to study because of the additional cancellation induced by the term $|t|^{-i \gamma}$, and that by letting $\gamma \rightarrow 0$, the properties of the Hilbert transform could be recovered. The first step in this program was successfully completed by Muckenhoupt [5] who showed that for each fixed $\gamma$,

$$
H_{\gamma} f=f \star \frac{1}{\pi} \frac{\operatorname{sgn} t}{|t|^{1+i \gamma}}
$$

exists, and that $H_{\gamma} f \rightarrow H_{o} f=\tilde{f}$ in $L^{2}$ norm. Later P. Ash [2] showed that $H_{\gamma} f \rightarrow H_{o} f=\tilde{f}$ in $L^{p}$ norm, $1<p<\infty$. Unfortunately in 1979 it was shown that $\lim _{\gamma \rightarrow 0^{+}} H_{\gamma} f(x)$ may fail to exist a.e. In [1] this is shown by construction of a function $f \in L^{p}(\mathbb{R})$, for all $p, 1<p<\infty$, such that

Received by the editors September 28, 1990.

1980 Mathematics Subject Classification (1985 Revision). Primary 42B20; Secondary 42A50, $42 \mathrm{~A} 45$.

Key words and phrases. Singular integral, Hilbert transform, multipliers, conjugate function, complex homogeneity.

The author was partially supported by NSF grant DMS-8910947. 
limsup $\sup _{\gamma \rightarrow 0^{+}} H_{\gamma} f(x)=\infty$ a.e. This example made use of the fact that the limit was being taken through a continuous parameter. In particular, for each fixed $x$, there was a set of $\gamma$ 's (of measure zero) such that $H_{\gamma} f(x)=\infty$. This left open the question of what happens if we use only a sequence $\left\{\gamma_{j}\right\}_{j=1}^{\infty}$ such that $\gamma_{j} \rightarrow 0$. In this paper we show that in fact for any sequence $\left\{\gamma_{j}\right\}_{j=1}^{\infty}$ such that $\lim _{j \rightarrow \infty} \gamma_{j}=0$, there exists a function $f \in L^{p}(\mathbb{R}), 1<p<\infty$, such that on a set of positive measure $\lim _{j \rightarrow \infty} H_{\gamma_{j}} f(x)$ fails to converge.

The operators given by convolution with $\frac{1}{\pi}\left(\operatorname{sgn} t /|t|^{1+i \gamma}\right)$ have Fourier multipliers given by $c_{\gamma} \operatorname{sgn}(\xi)|\xi|^{i \gamma}$ where $c_{\gamma}=2 \Gamma(-i \gamma) \sinh \left(\frac{\gamma \pi}{2}\right)$ is a constant that depends on $\gamma$ and converges to a finite nonzero limit as $\gamma \rightarrow 0$. (See $[2,5]$.)

\section{THE PERIODIC CASE}

We will first consider the easier case of $\mathbb{R}$ replaced by $T=[0,2 \pi)$. On $T$ we consider the operators given by the multipliers $c_{\gamma} \operatorname{sgn}(n)|n|^{i \gamma}$. The a.e. existence of these operators has been shown by Weiss and Zygmund [8]. These operators will be denoted by $\widetilde{T}_{\gamma}$, thus if $\varphi(x)=\sum \hat{\varphi}(n) e^{i n x}$ then $\widetilde{T}_{\gamma} \varphi(x)=$ $\sum \hat{\varphi}(n) c_{\gamma} \operatorname{sgn}(n)|n|^{i \gamma} e^{i n x}$.

Theorem 1. Let $\left\{\gamma_{j}\right\}_{j=1}^{\infty}$ be a sequence such that $\lim _{j \rightarrow \infty} \gamma_{j}=0$; then there exists a function $\varphi \in L^{\infty}(\mathbf{T})$ such that $\lim _{j \rightarrow \infty} \widetilde{T}_{\gamma_{j}} \varphi(x)$ fails to exist on a set of positive measure.

The proof will use Bourgain's Entropy method. The form of his theorem that will be most useful here is stated below. (See $[3,6]$ as well as Bourgain's origional paper [4] for additional applications of this useful result.)

Bourgain's Entropy Theorem [4, Proposition 2]. Let $(\Omega, \mu)$ denote a probability space. Assume $\left\{T_{n}\right\}_{n=1}^{\infty}$ is a sequence of uniformly bounded linear operators on $L^{2}(\Omega)$ and that these operators commute with a sequence $\left\{R_{j}\right\}$ of positive isometries that have $R_{j}(1)=1$ and satisfy $\frac{1}{J} \sum_{j=1}^{J} R_{j} f \rightarrow \int f d \mu$ in mean for $f \in L^{1}(\Omega)$. Under these conditions, if there is a number $\beta>0$ such that for each $N \geq 1$ there is a function $\varphi$ (which is allowed to depend on $N$ ), $\|\varphi\|_{2} \leq 1$, and a collection $\left\{T_{n_{i}}\right\}_{i=1}^{N}$ such that $\left\|T_{n_{i}} \varphi-T_{n_{j}} \varphi\right\|_{2}>\beta$ for each $i \neq j, i=1, \ldots, N, j=1, \ldots, N$, then there is bounded function $\varphi$ such that $\left\{T_{n} \varphi\right\}$ is not a.e. convergent.

In our application we will let $\Omega=[0,2 \pi), \mu$ be the normalized Lebesque measure, $T_{n}=\widetilde{T}_{\gamma_{n}} / c_{\gamma_{n}}$ and $R_{j}(x)=x+j \alpha \bmod 2 \pi$, where $\alpha$ can be any real number not rationally related to $2 \pi$. Since we know that $\lim _{n \rightarrow \infty} c_{\gamma_{n}}$ exists and is nonzero, if we can show that $\lim _{n \rightarrow \infty} T_{n}$ fails to exist, we can conclude that $\lim _{n \rightarrow \infty} \widetilde{T}_{\gamma_{n}}$ fails to exist.

The required function $\varphi$ will have the form

$$
\varphi(x)=\frac{1}{\sqrt{L}} \sum_{n=1}^{L} e^{i 2^{n} x},
$$

where $L$ will be determined later. Note that $\|\varphi\|_{2}=1$. For this function, we have

$$
\widetilde{T}_{\gamma} \varphi(x)=\frac{1}{\sqrt{L}} \sum_{n=1}^{L} c_{\gamma}\left(\operatorname{sgn} 2^{n}\right) e^{i \gamma \cdot n \cdot \ln 2} e^{i 2^{n} x} .
$$


Consequently,

$$
T_{r} \varphi(x)-T_{s} \varphi(x)=\frac{1}{\sqrt{L}} \sum_{n=1}^{L}\left(\operatorname{sgn} 2^{n}\right) e^{i 2^{n} x} e^{i \gamma_{r} \cdot n \cdot \ln 2}\left(1-e^{i\left(\gamma_{s}-\gamma_{r}\right) n \cdot \ln 2}\right),
$$

and thus

$$
\begin{aligned}
\left\|T_{r} \varphi-T_{s} \varphi\right\|_{2}^{2} & =\frac{1}{L} \sum_{n=1}^{L}\left|1-e^{i\left(\gamma_{s}-\gamma_{r}\right) n \cdot \ln 2}\right|^{2} \\
& =\frac{1}{L} \sum_{n=1}^{L} 2\left(1-\cos \left[\left(\gamma_{s}-\gamma_{r}\right) n \cdot \ln 2\right]\right) .
\end{aligned}
$$

Let $\theta_{s r}=\frac{1}{2 \pi}\left(\gamma_{s}-\gamma_{r}\right) \ln 2$, and define $\Omega=\left\{\theta_{s r}: 1 \leq r<s \leq N\right\}$. We need to find an $L$ such that for each $\theta \in \Omega$, we have $\frac{1}{L} \sum_{n=1}^{L} 2(1-\cos 2 \pi n \theta)>\beta$. Let $\Omega=\Omega_{1} \cup \Omega_{2}$ where

$$
\Omega_{1}=\{\theta: \theta \in \Omega, \theta \text { irrational }\} \text { and } \Omega_{2}=\{\theta: \theta \in \Omega, \theta \text { rational }\} .
$$

There are two cases. If $\theta \in \Omega_{1}$, we know that $\{n \theta\}$ is uniformly distributed mod 1 , and consequently, since $(1-\cos 2 \pi x)$ is Riemann integrable and $\int_{0}^{1} 2(1-\cos 2 \pi x) d x=2$, there exists an $l=l(\theta)$ such that

$$
\frac{1}{L} \sum_{n=1}^{L} 2(1-\cos 2 \pi n \theta)>\left(\frac{1}{2}\right) 2=1
$$

for all $L>1$. There are only a finite number of numbers in $\Omega_{1}$, we let $L_{1}=\max _{\theta \in \Omega_{1}} l(\theta)$.

If $\theta \in \Omega_{2}$ then $\theta$ is rational; we need to prove a similar inequality. Assume $\theta=p / q$, where $p$ and $q$ are integers and $(p, q)=1$. Then we have $\{n \theta\}=$ $\{n p / q\}$. If $L$ is a multiple of $q$, then the points $\{n p / q\}_{n=1}^{L}$ are uniformly distributed in the set $\{k / q\}_{k=0}^{q-1}$. Thus at least $1 / 4$ of the points will be a distance of at least $1 / 4$ away from zero. Since $1-\cos (2 \pi x) \geq 1$ for $1 / 4 \leq$ $x \leq 3 / 4$, we have

$$
\frac{1}{L} \sum_{n=1}^{L} 2(1-\cos 2 \pi n \theta) \geq \frac{1}{L}\left(\frac{L}{4}\right) 2(1)=\frac{1}{2} .
$$

Now let $L_{2}$ be the product $q_{1} q_{2} \cdots q_{j}$ where $\left\{p_{i} / q_{i}: i=1, \ldots, j\right\}$ represent the rationals in $\Omega_{2}$. Finally let $L=L_{1} L_{2}$. We see that conditions are satisfied for both estimates above, and hence we have $\left\|T_{r} \varphi-T_{s} \varphi\right\|_{2}^{2} \geq 1 / 2$ for $r=$ $1,2, \ldots, N, s=1,2, \ldots, N, r \neq s$ as desired.

Corollary. For any $p, 1<p \leq 2$, and any $B>0$, there exists a function $\varphi=\varphi_{B}$ in $L^{p}(\mathbf{T})$ such that

$$
\left|\left\{x: \sup _{n}\left|\widetilde{T}_{\gamma_{n}} \varphi(x)\right|>\lambda\right\}\right|>\frac{B}{\lambda^{p}}\|\varphi\|_{p}^{p}
$$

for some $\lambda>0$. In addition, $\varphi$ can be taken to be a trigonometric polynomial. Proof. By Theorem 1, we know that we do not have convergence for some $\varphi \in L^{\infty}(\mathbf{T})$ and hence for some $\varphi \in L^{p}(\mathbf{T})$. By Stein's theorem [7], this 
implies the failure of a weak type $(p, p)$ inequality for $1<p \leq 2$. Because trigonometric polynomials are dense, if we had a weak type inequality for all trigonometric polynomials we would have it for all $L^{p}(\mathbf{T})$.

\section{THE NONCOMPACT CASE}

We also can state a version of Theorem 1 for the real line.

Theorem 2. Let $\left\{\gamma_{j}\right\}_{j=1}^{\infty}$ be a sequence such that $\lim _{n \rightarrow \infty} \gamma_{n}=0$, then there exists a function $f \in L^{p}(\mathbb{R}), 1<p \leq \infty$, such that $\lim _{n \rightarrow \infty} H_{\gamma_{n}} f(x)$ fails to exist on a set of positive measure.

Proof. Bourgain's Entropy Theorem requires operators to act on a probability space. Thus we cannot use his method to directly give a counterexample on $\mathbb{R}$. However we will take advantage of our example on $\mathbf{T}$. See [1] where a similar idea is used. Some steps in the computation below are given in greater detail in [1].

Let $\varphi$ be a function in $L^{\infty}(\mathbf{T})$ such that $\lim _{n \rightarrow \infty} \widetilde{T}_{\gamma_{n}} \varphi(x)$ fails to exist on a set $E$, with $|E|>0$. Such a function exists by Theorem 1 . Write $\varphi(x)=$ $\sum_{k=-\infty}^{\infty} a_{k} e^{i k x}$. We can assume without loss of generality that $\int_{-\pi}^{\pi} \varphi(x) d x=0$, and hence that $a_{o}=0$. Define a function $f$ on $\mathbb{R}$ by

$$
\hat{f}(\xi)=\sum_{k=-\infty}^{\infty} a_{k} \chi_{(-k-1 / 2,-k+1 / 2)}(\xi) \text {. }
$$

Thus

$$
\left(H_{\gamma} f\right)^{\wedge}(\xi)=\sum_{k=-\infty}^{\infty} a_{k} \chi_{(-k-1 / 2,-k+1 / 2)}(\xi) c_{\gamma} \operatorname{sgn}(\xi)|\xi|^{i \gamma} .
$$

Taking inverse Fourier transforms, we have

$$
\begin{aligned}
H_{\gamma} f(x)= & \frac{1}{2 \pi} \int_{-\infty}^{\infty}\left(H_{\gamma} f\right)^{-}(\xi) e^{-i \xi x} d \xi \\
= & \frac{1}{2 \pi} \int_{-\infty}^{\infty} \sum_{k=-\infty}^{\infty} a_{k} \chi_{(-k-1 / 2,-k+1 / 2)}(\xi) c_{\gamma} \operatorname{sgn}(\xi)|\xi|^{i \gamma} e^{-i \xi x} d \xi \\
= & \frac{1}{2 \pi} \sum_{k=-\infty}^{\infty} a_{k} \int_{-k-1 / 2}^{-k+1 / 2} c_{\gamma} \operatorname{sgn}(\xi)|\xi|^{i \gamma} e^{-i \xi x} d \xi \\
= & \frac{1}{2 \pi} \sum_{k=-\infty}^{\infty} a_{k} c_{\gamma}\left[\int_{-k-1 / 2}^{-k+1 / 2} c_{\gamma} \operatorname{sgn}(\xi)|k|^{i \gamma} e^{-i \xi x} d \xi\right. \\
& \left.\quad+\int_{-k-1 / 2}^{-k+1 / 2} \operatorname{sgn}(\xi)\left(|\xi|^{i \gamma}-|k|^{i \gamma}\right) e^{-i \xi x} d \xi\right] \\
= & \frac{1}{2 \pi} \sum_{k=-\infty}^{\infty} a_{k} c_{\gamma} \operatorname{sgn}(k)|k|^{i \gamma} e^{i k x}\left(\frac{\sin (x / 2)}{x / 2}\right)+\varepsilon(x, \gamma),
\end{aligned}
$$

where $\varepsilon(x, \gamma)=\frac{1}{2 \pi} \sum_{k=-\infty}^{\infty} a_{k} c_{\gamma} \int_{-k-1 / 2}^{-k+1 / 2} \operatorname{sgn}(\xi)\left(|\xi|^{i \gamma}-|k|^{i \gamma}\right) e^{-i \xi x} d \xi$. Thus

$$
H_{\gamma} f(x)=\left(\frac{\sin (x / 2)}{x / 2}\right) \widetilde{T}_{\gamma} \varphi(x)+\varepsilon(x, \gamma) .
$$


However,

$$
|\varepsilon(x, \gamma)| \leq \frac{\left|c_{\gamma}\right|}{2 \pi} \sum_{k=-\infty}^{\infty}\left|a_{k}\right| \int_{-k-1 / 2}^{-k+1 / 2} \|\left.\xi\right|^{i \gamma}-|k|^{i \gamma} \mid d \xi .
$$
by

By the mean value theorem, we can estimate the integral in this expression

$$
\sup _{\xi \in(k-1 / 2, k+1 / 2)}\left|\left(e^{i \gamma \ln \xi}\right)^{\prime}\right| \frac{1}{2} \leq \frac{\gamma}{|k|-1 / 2}\left(\frac{1}{2}\right) .
$$

Consequently,

$$
\begin{aligned}
|\varepsilon(x, \gamma)| & \leq \frac{\left|c_{\gamma}\right|}{4 \pi} \sum_{k=-\infty}^{\infty}\left|a_{k}\right| \frac{\gamma}{|k|-1 / 2} \\
& \leq \gamma \frac{\left|c_{\gamma}\right|}{4 \pi}\left(\sum_{k=-\infty}^{\infty}\left|a_{k}\right|^{2}\right)^{1 / 2}\left(\sum_{k=-\infty}^{\infty} \frac{1}{(|k|-1 / 2)^{2}}\right)^{1 / 2}=O(\gamma) .
\end{aligned}
$$

We now know

$$
H_{\gamma} f(x)=\left(\frac{\sin (x / 2)}{x / 2}\right) \tilde{T}_{\gamma} \varphi(x)+\varepsilon(x, \gamma), \quad \text { with } \varepsilon(x, \gamma)=O(\gamma)
$$

For each $x \in E$, we know $\lim _{n \rightarrow \infty} \widetilde{T}_{\gamma_{n}} \varphi(x)$ will fail to exist. Since $\lim _{n \rightarrow \infty} \varepsilon(x, \gamma)=0$, we know that $\lim _{n \rightarrow \infty} H_{\gamma_{n}} f(x)$ must also fail to exist on $E$.

It remains to show that $f \in L^{p}(\mathbb{R}), 1<p \leq \infty$. To see this we compute $f$ explicitly, and find that

$$
f(x)=\frac{1}{2 \pi} \sum_{k=-\infty}^{\infty} a_{k} e^{i k x}\left(\frac{\sin x / 2}{x / 2}\right) .
$$

Consequently

$$
\|f\|_{p}^{p}=\left(\frac{1}{2 \pi}\right)^{p} \sum_{n=-\infty}^{\infty} \int_{2 \pi n}^{2 \pi(n+1)}\left|\frac{\sin x / 2}{x / 2}\right|^{p}\left|\sum_{k=-\infty}^{\infty} a_{k} e^{i k x}\right|^{p} d x .
$$

First note that $\left|\frac{\sin x / 2}{x / 2}\right|$ is bounded by $2 / n$ on $[2 \pi n, 2 \pi(n+1)]$ if $n \neq 0$, or -1 , and by 1 on $[-2 \pi, 0]$ and $[0,2 \pi]$. Also note that

$$
\int_{2 \pi n}^{2 \pi(n+1)}\left|\sum_{k=-\infty}^{\infty} a_{k} e^{i k x}\right|^{p} d x
$$

is a constant $b_{p}$ independent of $n$. Consequently

$$
\|f\|_{p}^{p} \leq\left(\frac{1}{2 \pi}\right)^{p}\left(2+\sum_{\substack{n=-\infty \\ n \neq-1,0}}^{\infty} \frac{2^{p}}{|n|^{p}}\right) b_{p}<\infty .
$$

Remark. Theorem 2 can be strengthened to give a function that is in all $L^{p}(\mathbb{R})$, $0<p \leq \infty$, such that almost everywhere convergence of $\left\{H_{\gamma_{n}} f\right\}$ fails. The idea is to replace translates of the characteristic function of $(-1 / 2,1 / 2)$ by a suitably smooth "bump function." See [1] for details. 


\section{REFERENCES}

1. J. M. Ash, P. Ash, C. Fefferman, and R. L. Jones, Singular integral operators with complex homogeneity, Studia Math. 65 (1979), 31-50.

2. P. Ash, On singular integrals with complex homogeneity, Ph.D Thesis, Univ. of Illinois, Chicago, Illinois.

3. A. Bellow, R. L. Jones, and J. Rosenblatt, Almost everywhere convergence of convolution powers, preprint.

4. J. Bourgain, Almost sure convergence and bounded entropy, Israel J. Math 62 (1988), 79-97.

5. B. Muckenhoupt, On certain singular integrals, Pacific J. Math. 10 (1961), 239- 291.

6. J. Rosenblatt, Universally bad sequences in ergodic theory, Almost Everywhere Convergence II, (Proc. of the Conference on Almost Everywhere Convergence in Probability and Ergodic Theory, Evanston, IL, Oct 16-20) (A. Bellow and R. Jones, eds.), Academic Press, 1991, pp. 223-242.

7. E. M. Stein, On limits of sequences of operators, Ann. of Math. (2) 74 (1961), 140- 170.

8. M. Weiss and A. Zygmund, On multipliers preserving convergence of trigonometric series almost everywhere, Studia Math. 30 (1968), 111-120.

Department of Mathematics, DePaul University, Chicago, Illinois 60614 